\title{
Nucleon-Nucleon Chiral Two Pion Exchange potential vs Coarse grained interactions
}

\section{Rodrigo Navarro Perez ${ }^{* i}$}

Departamento de Física Atómica, Molecular y Nuclear and Instituto Carlos I de Física Teórica y Computacional. Universidad de Granada, E-18071 Granada, Spain.

E-mail: rnavarropeugr.es

\section{J. E. Amaro}

Departamento de Física Atómica, Molecular y Nuclear and Instituto Carlos I de

Física Teórica y Computacional. Universidad de Granada, E-18071 Granada, Spain.

E-mail: amaro@ugr.es

\section{E. Ruiz Arriola}

Departamento de Física Atómica, Molecular y Nuclear and Instituto Carlos I de Física Teórica y Computacional. Universidad de Granada, E-18071 Granada, Spain.

E-mail: earriola@ugr.es

We analyse the interplay between nucleon-nucleon potentials deduced from chiral perturbation theory and a coarse grained representation of the short distance interactions by delta-shells potentials below a certain cut-off distance. While we find that the number of parameters is greatly reduced when Chiral Two Pion Exchange contributions are included we also observe that discerning the necessity of improvements on the interaction requires a detailed analysis of all error sources. Our points are best illustrated by computing deuteron static properties as well as electromagnetic form factors after error propagation.

The 7th International Workshop on Chiral Dynamics,

August 6 -10, 2012

Jefferson Lab, Newport News, Virginia, USA

\footnotetext{
${ }^{*}$ Speaker.

${ }^{\dagger}$ Work supported by Spanish DGI (grant FIS2011-24149) and Junta de Andalucía (grant FQM225). R.N.P. is supported by a Mexican CONACYT grant.
} 


\section{Introduction}

The chiral theory of Nuclear Forces has become a popular approach in recent years $[1,2]$. Indeed, while Charge Dependence and One Pion Exchange provided a satisfactory fit to $\mathrm{np}$ and $\mathrm{pp}$ data [3] leading to high quality potentials used for Nuclear applications [3, 4, 5, 6], Chiral Two Pion Exchange potentials [7] have improved the analysis [8,9].

In this contribution we reanalyze the problem directly in terms of a delta-shell potential which gives a simple way to coarse grain the interaction between two nucleons down to the relevant shortest de Broglie wavelength [10]. This form was first introduced by Aviles [11] and has recently been used to calculate nuclear binding energies [10], to extract [12] and propagate [13,14] the corresponding uncertainties inherent to the NN interaction or to evaluate the effective interactions [15].

\section{Delta-shell and Chiral Potentials}

In our analysis the potential consists of a short range piece and a long range contribution as follows

$$
V(r)=\sum_{n=1}^{18} O_{n}\left[\sum_{i} V_{i, n} r_{i} \delta\left(r-r_{i}\right)\right]+\left[V_{\mathrm{OPE}}(r)+V_{\mathrm{TPE} 1 \mathrm{o}}(r)+V_{\mathrm{TPEso}}(r)+V_{\mathrm{em}}(r)\right] \theta\left(r-r_{c}\right),
$$

where $O_{n}$ are the set of operators in the AV18 basis [4], $r_{i}$ are the concentration radii and $V_{i, n}$ are strength coefficients, which are used as fitting parameters. For definiteness $V_{\mathrm{OPE}}(r), V_{\mathrm{TPElo}}(r)$, $V_{\text {TPEso }}(r)$ and $V_{\mathrm{em}}(r)$ are those of Ref. [8]. The distance between the delta-shells $\Delta r$ is determined from the shortest de Broglie wavelength (for a detailed explanation see the appendix in [16] and [10]) below pion production threshold i.e. $\Delta r=1 / \sqrt{M_{N} m_{\pi}} \sim 0.6 \mathrm{fm}$, so that $r_{i}=i \Delta r \leq r_{c}$. Our purpose is to see how small can $r_{c}$ become when $V_{\mathrm{OPE}}(r), V_{\mathrm{TPElo}}(r)$ and $V_{\mathrm{TPEso}}(r)$ in Eq. (2.1) are subsequently added.

\section{Chiral TPE vs OPE}

As a preliminary step in our analysis we fitted Eq. (2.1) to a pseudo-database constructed from the np phase-shifts given by the 1993 Partial Wave Analysis and the subsequent phase-shifts of the 6 high quality potentials that give a $\chi^{2} / v \lesssim 1$ when compared to experimental scattering data $[3,4,5,6]$. Given this, we have two alternatives based on the treatment of this pseudodata. Either we make accurate fits to each single potential phase-shifts and we average the seven different results and determine their mean squared deviation or we asign a mean value and an error to the compilation as a whole and make a standard fit taking the pseudodata with errors as experimental data. While the first procedure seems to be a quite natural way to incorporate correlations between different partial waves, it turns out that these correlations are almost negligible as can be seen for some representative cases in Fig. (1).

Table (1) shows the value of $\chi^{2} / v$ and the number of parameters for every potential depending on the long range interaction and the radial cut-off. The results show, in agreement with previous findings, that for OPE and TPElo the range of validity is between $1.8 \mathrm{fm}$ and $2.4 \mathrm{fm}$ since using 

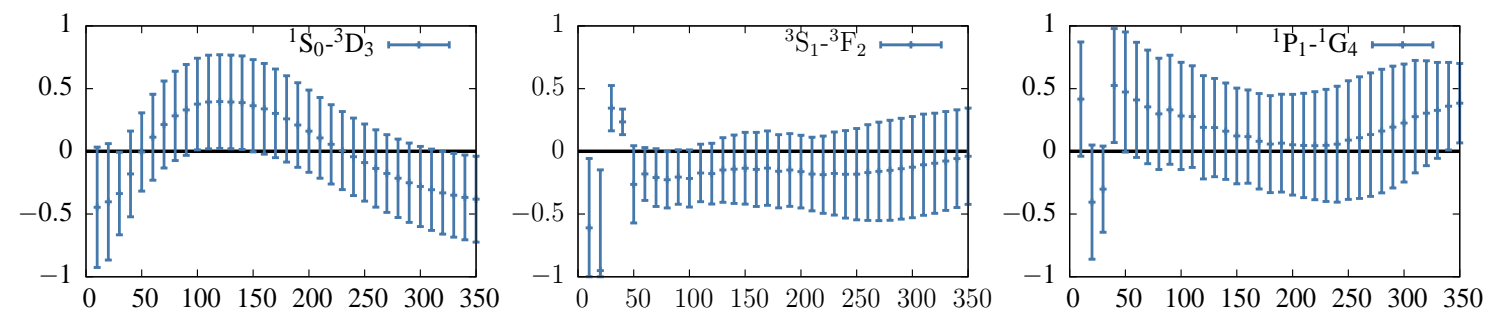

Figure 1: Correlations among different phase-shifts of the PWA and six high quality potentials $[3,4,5,6]$ which provided a $\chi^{2} /$ d.o.f $\lesssim 1$. The correlation factor was calculated using the equation $r_{x, y}=$ $\sum_{i=1}^{n}\left(x_{i}-\bar{x}\right)\left(y_{i}-\bar{y}\right) /\left(n \sigma_{x} \sigma_{y}\right)$ where the bar indicates the mean of a variable and $\sigma$ the corresponding standard deviation.

\begin{tabular}{ccccccc}
$r_{\mathrm{c}}[\mathrm{fm}]$ & 1.8 & & 2.4 & \multicolumn{3}{c}{3.0} \\
\hline & $\# \mathrm{p}$ & $\chi^{2} / v$ & $\# \mathrm{p}$ & $\chi^{2} / v$ & $\# \mathrm{p}$ & $\chi^{2} / v$ \\
\hline OPE & 37 & 2.1383 & 47 & 0.6470 & 51 & 0.4653 \\
TPElo & 40 & 2.0661 & 46 & 0.7361 & 52 & 0.5047 \\
TPEso & 32 & 0.5911 & 44 & 0.5225 & 51 & 0.3928
\end{tabular}

Table 1: $\chi^{2} / v$ and number of parameters for fits to phasehifts

\begin{tabular}{cccc}
$r_{\mathrm{c}}[\mathrm{fm}]$ & 1.8 & 2.4 & 3.0 \\
& $\chi^{2} / v$ & $\chi^{2} / v$ & $\chi^{2} / v$ \\
\hline OPE & 2.45 & 0.56 & 0.47 \\
TPElo & 2.92 & 0.69 & 0.49 \\
TPEso & 0.54 & 0.70 & 0.41
\end{tabular}

Table 2: $\chi^{2} / v$ to wolfenstein parameters

$r_{c}=1.8 \mathrm{fm}$ no longer gives a satisfactory fit. With TPEso one can go down to $r_{c}=1.8 \mathrm{fm}$ and even reduce the number of parameters needed.

Just as the PWA and the 6 high quality potential show a dispersion on the phaseshifts the same occurs with the scattering amplitude. This can be easily seen by using the Wolfenstein decomposition of the scattering amplitude [17],

$$
\begin{aligned}
M\left(\mathbf{k}_{f}, \mathbf{k}_{i}\right)= & a(\boldsymbol{\theta}, p)+m(\boldsymbol{\theta}, p)\left(\sigma_{1}, \mathbf{n}\right)\left(\sigma_{2}, \mathbf{n}\right)+(g(\boldsymbol{\theta}, p)-h(\boldsymbol{\theta}, p))\left(\sigma_{1}, \mathbf{m}\right)\left(\sigma_{2}, \mathbf{m}\right) \\
& +(g(\boldsymbol{\theta}, p)+h(\boldsymbol{\theta}, p))\left(\sigma_{1}, \mathbf{l}\right)\left(\sigma_{2}, \mathbf{l}\right)+c(\theta, p)\left(\sigma_{1}+\sigma_{2}, n\right),
\end{aligned}
$$

and comparing the 5-complex Wolfenstein parameters for every interaction. Since all the scattering observables can be directly calculated from the Wolfenstein parameters the dispersion on the amplitude can be a measure of the dispersion on observables as well. With this in mind we calculated the Wolfenstein parameters of the potentials in table (1) as a function of laboratory energy $T_{\mathrm{LAB}}$ and scattering angle $\theta$ and compared them to the mean of the PWA and 6 high quality potentials using the standard deviation as the uncertainty to calculate a $\chi^{2} / v$. The results are shown in table (2) and exhibit very similar features to the ones in table (1). Figure (2) shows the disperssion of the Wolfenstein parameters for the high quality potentials as a function of the scattering angle $\theta$ 

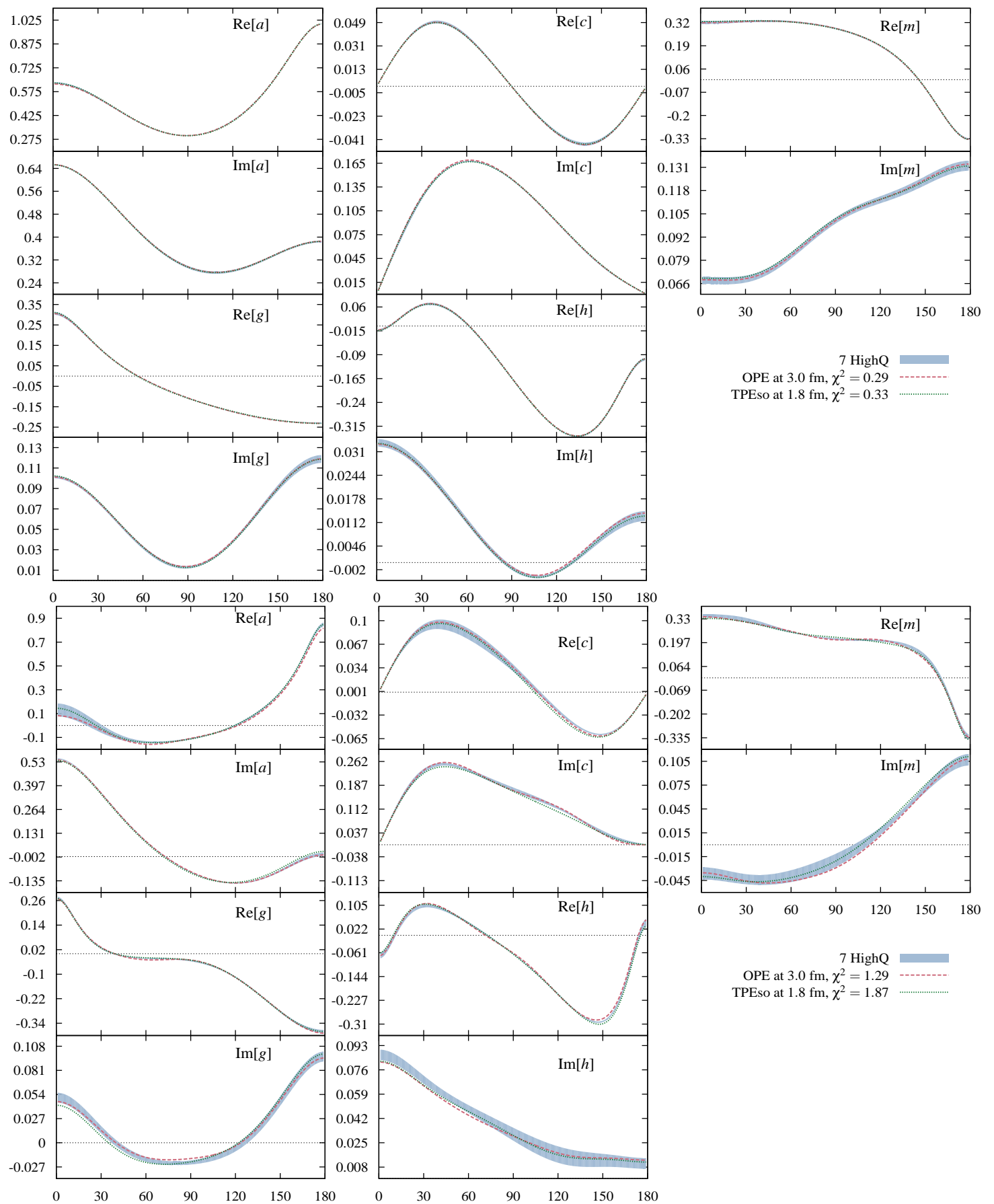

Figure 2: $\mathrm{np}$ Wolfenstein parameters for different energies in the laboratory system as a function of the $\mathrm{CM}$ angle. Upper panel: $E_{\mathrm{LAB}}=100 \mathrm{MeV}$. Lower panel: $E_{\mathrm{LAB}}=350 \mathrm{MeV}$. The band represents the compilation of the PWA and six high quality potentials $[3,4,5,6]$ which provided a $\chi^{2} /$ d.o.f $\lesssim 1$. The dashed line denotes the results obtained by the fitted interaction with OPE and $r_{c}=3.0 \mathrm{fm}$, while the doted line comes from the interaction with TPE and $r_{c}=1.8 \mathrm{fm}$. 


\begin{tabular}{cccccccc} 
Potential & $r_{c}(\mathrm{fm})$ & $B_{D}(\mathrm{MeV})$ & $\eta$ & $A_{S}\left(\mathrm{fm}^{1 / 2}\right)$ & $P_{D}$ & $r_{m}(\mathrm{fm})$ & $Q_{D}\left(\mathrm{fm}^{2}\right)$ \\
\hline OPE & 3.0 & $-2.2(2)$ & $0.025(2)$ & $0.88(3)$ & $5.7(2)$ & $1.97(8)$ & $0.272(9)$ \\
TPElo & 2.4 & $-2.2(3)$ & $0.025(2)$ & $0.89(4)$ & $5.6(3)$ & $2.0(1)$ & $0.27(1)$ \\
TPEso & 1.8 & $-2.2(4)$ & $0.025(3)$ & $0.88(6)$ & $5.6(4)$ & $2.0(1)$ & $0.27(2)$ \\
\hline Empirical & & $-2.2245(2)$ & $0.0256(5)$ & $0.8781(44)$ & $5.67(4)$ & $1.953(3)$ & $0.2859(3)$
\end{tabular}

Table 3: Deuteron properties. Notation is as follows, $B_{D}$ binding energy, $\eta$ assymptotic ratio, $A_{S} \mathrm{~S}$-state normalization, $P_{D}$ D-state probability, $r_{m}$ root mean square radius and $Q_{D}$ electric quadrupole moment.

at $T_{\mathrm{LAB}}=100 \mathrm{MeV}$ and $T_{\mathrm{LAB}}=350 \mathrm{MeV}$. We also show the Wolfenstein parameters given by the coarse grained interactions with OPE using $r_{c}=3.0 \mathrm{fm}$ and TPEso with $r_{c}=1.8 \mathrm{fm}$.

\section{Deuteron Properties}

For a comparison between OPE and (chiral) TPE we calculate a few deuteron properties with the potentials constructed in this contribution. The results are shown in table (3) and show no significant diference on the central values and very similar uncertainties, being all of them compatible with previously known empirical values, and reflecting the pseudodata uncertainties.

Deuteron form factors using OPE with $r_{c}=3.0 \mathrm{fm}$ and TPElo with $r_{c}=1.8 \mathrm{fm}$ are presented Fig. (3) with propagated uncertainties. As we see there is no significant difference between using OPE or TPE as the long range np interaction. The rather small discrepancy between calculated and experimental values could be resolved by the inclusion of Meson Exchange Currents (MEC). In the $G_{C}$ form factor we see that within errors there is no discrepancy.

\section{Conclusions}

In the present contribution we have adressed a comparison between the well-known OPE potential and the chiral TPE interactions. The short distance piece of the potential is represented by a delta-shells potential which features a coarse graining of the unknown physics down to the smallest de Broglie wavelength probed by the NN interactions below pion production threshold.
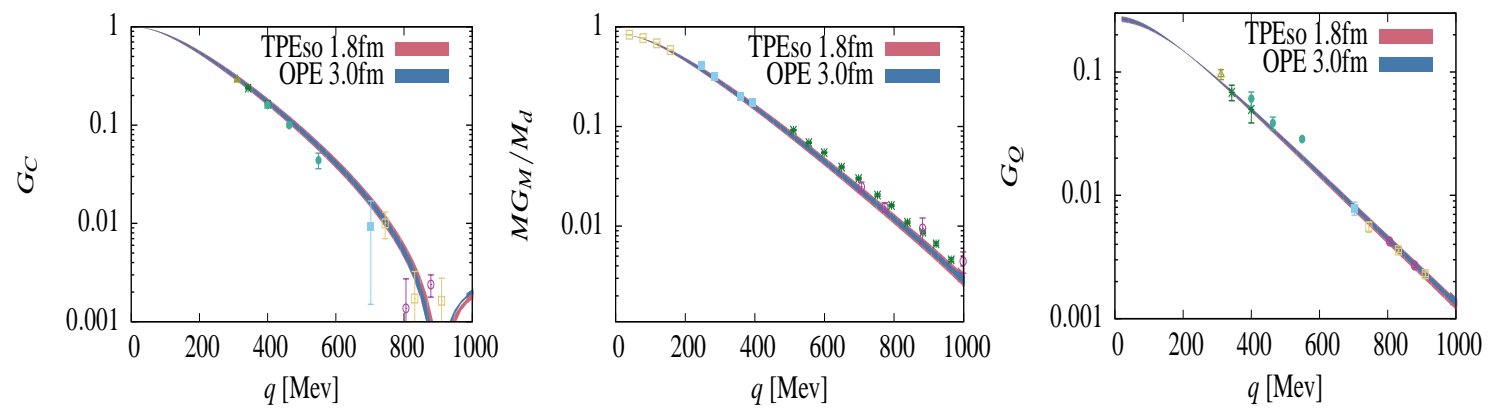

Figure 3: Deuteron Form Factors from OPE with $r_{c}=3.0 \mathrm{fm}$ (blue band) and TPE with $r_{c}=1.8 \mathrm{fm}$ (red band). The error bar was obtained by propagating the uncertainty from the pseudodata as explained in the text. 
The long range part is assumed to be valid down to a radial cut-off distance $r_{c}$ and we analyze the quality of each fit as a function of this distance. For our analysis we use pseudodata consisting of a compilation of the np phase shifts given by 7 high quality fits $[3,4,5,6]$. The error asignment corresponds to a lack of correlation between different partial waves; a circumstance which turns out to be true within the inherent uncertainties of the different potentials. There is substantial reduction in the number of parameters needed for the short range part of the interaction. Indeed for OPE, one has $r_{c}=3.0 \mathrm{fm}, \chi^{2} / v=0.47$ and 51 parameters are needed whilst OPE + (chiral) TPEso, gives $r_{c}=1.8 \mathrm{fm}, \chi^{2} / v=0.59$ and the number of parameters is reduced to 32. From a Nuclear Physics Structure point of view it is uncertain what could be the real advantage in implementing as a matter of principle the chiral TPE interaction. Actually, to decide objectively on this issue requires a meticulous determination of both statistical and systematic errors. We have illustrated this point by computing the deuteron form factors and propagating the corresponding uncertainties deduced by the error treatment of the pseudodata. This is a crucial issue to discern on the real role of the MEC conributions to the form factors. For instance, the charge form factor acquires purely transverse contributions which have been estimated to be small. The question is whether or not the size of the MEC's is larger than the estimated uncertainties.

\section{References}

[1] Evgeny Epelbaum, Hans-Werner Hammer, and Ulf-G. Meissner. Rev.Mod.Phys., 81:1773-1825, 2009.

[2] R. Machleidt and D.R. Entem. Phys.Rept., 503:1-75, 2011.

[3] V. G. J. Stoks, R. A. M. Kompl, M. C. M. Rentmeester, and J. J. de Swart. Phys. Rev., C48:792-815, 1993.

[4] Robert B. Wiringa, V. G. J. Stoks, and R. Schiavilla. Phys. Rev., C51:38-51, 1995.

[5] R. Machleidt. Phys. Rev., C63:024001, 2001.

[6] Franz Gross and Alfred Stadler. Phys.Rev., C78:014005, 2008.

[7] Norbert Kaiser, R. Brockmann, and W. Weise. Nucl.Phys., A625:758-788, 1997.

[8] M. C. M. Rentmeester, R. G. E. Timmermans, James Lewis Friar, and J. J. de Swart. Phys. Rev. Lett., 82:4992-4995, 1999.

[9] M.C.M. Rentmeester, R.G.E. Timmermans, and J. J. de Swart. Phys.Rev., C67:044001, 2003.

[10] R. Navarro Perez, J.E. Amaro, and E. Ruiz Arriola. Prog.Part.Nucl.Phys., 67:359-364, 2012.

[11] J. B. Aviles. Phys. Rev., C6:1467-1484, 1972.

[12] R. Navarro Perez, J. E. Amaro and E. Ruiz Arriola, arXiv:1202.2689 [nucl-th].

[13] R. Navarro Perez, J. E. Amaro and E. Ruiz Arriola, arXiv:1202.6624 [nucl-th].

[14] R. Navarro Perez, J.E. Amaro, and E. Ruiz Arriola. PoS, QNP2012:145, 2012.

[15] R. Navarro Perez, J. E. Amaro and E. Ruiz Arriola, arXiv:1209.6269 [nucl-th].

[16] D.R. Entem, E. Ruiz Arriola, M. Pavon Valderrama, and R. Machleidt. Phys.Rev., C77:044006, 2008.

[17] J. Golak, W. Glockle, R. Skibinski, H. Witala, D. Rozpedzik, et al. Phys.Rev., C81:034006, 2010. 